\title{
Fecal Assays Detect Hypersensitivity to Cow's Milk Protein and Gluten in Adults With Irritable Bowel Syndrome
}

\author{
ANTONIO CARROCCIO, * IGNAZIO BRUSCA, ${ }^{\ddagger}$ PASQUALE MANSUETO, ${ }^{\S}$ MAURIZIO SORESI, ${ }^{\S}$ ALBERTO D’ALCAMO, ${ }^{\S}$ \\ GIUSEPPE AMBROSIANO,§ ILENIA PEPE, ${ }^{\S}$ GIUSEPPE IACONO," MARIA LETIZIA LOSPALLUTI," STELLA M. LA CHIUSA, \\ and GAETANA DI FEDE?
}

*Internal Medicine, Ospedali Civili Riuniti di Sciacca (AG), and University of Palermo; " Clinical Chemistry, "Buccheri La Ferla" Hospital, Palermo; \$Internal Medicine, Department of Clinical Medicine and Emerging Diseases, University Hospital of Palermo; "Pediatric Gastroenterology, "Di Cristina" Hospital, Palermo; and

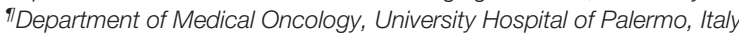

BACKGROUND \& AIMS: Some patients with irritable bowel syndrome (IBS)-like symptoms suffer from food hypersensitivity $(\mathrm{FH})$; their symptoms improve when they are placed on elimination diets. No assays identify patients with $\mathrm{FH}$ with satisfactory levels of sensitivity. We determined the frequency of FH among patients with symptoms of IBS and the ability of fecal assays for tryptase, eosinophil cationic protein (ECP), or calprotectin to diagnose FH. METHODS: The study included 160 patients with IBS, 40 patients with other gastrointestinal diseases, and 50 healthy individuals (controls). At the start of the study, patients completed a symptom severity questionnaire, fecal samples were assayed, and levels of specific immunoglobulin E were measured. Patients were observed for 4 weeks, placed on an elimination diet (without cow's milk and derivatives, wheat, egg, tomato, and chocolate) for 4 weeks, and kept a diet diary. Those who reported improvements after the elimination diet period were then diagnosed with $\mathrm{FH}$, based on the results of a double-blind, placebo-controlled, oral food challenge (with cow's milk proteins and then with wheat proteins). RESULTS: Forty of the patients with IBS (25\%) were found to have FH. Levels of fecal ECP and tryptase were significantly higher among patients with IBS and FH than those without FH. The ECP assay was the most accurate assay for diagnosis of $\mathrm{FH}$, showing $65 \%$ sensitivity and $91 \%$ specificity. CONCLUSIONS: Twenty-five percent of patients with IBS have FH. These patients had increased levels of fecal ECP and tryptase, indicating that they might cause inflammation in patients with IBS. Fecal assays for ECP could be used to identify FH in patients with IBS.

Keywords: Cow's Milk Protein Hypersensitivity; Gluten Sensitivity; Fecal Tryptase; Fecal Eosinophil Cationic Protein; Fecal Calprotectin.

Watch this article's video abstract and others at http:// tiny.cc/bz9jv.

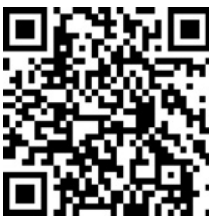

Scan the quick response (QR) code to the left with your mobile device to watch this article's video abstract and others. Don't have a QR code reader? Get one at mobiletag.com/en/download.php.
$\mathrm{I}_{\mathrm{r}}^{\mathrm{r}}$ rritable bowel syndrome (IBS) is a common gastrointestinal disorder which accounts for about $40 \%$ of all gastroenterology outpatient consultations. ${ }^{1}$ Although an alteration of the brain-gut axis is considered to have a fundamental role, ${ }^{2}$ gut mucosal inflammation is certainly important in the complex pathogenesis of IBS. $^{3}$ In fact, several studies have shown an increased number of immunocytes both in the lamina propria and in the epithelium of mucosal biopsy specimens from IBS patients. ${ }^{4-10}$ Lamina propria and intraepithelial lymphocytes, ${ }^{5,10}$ mast cells, ${ }^{4,7,9}$ and macrophages ${ }^{11}$ are among the immunocytes involved in mucosal inflammation in IBS, and a role for a previous infectious enteritis in determining this inflammation has been established. ${ }^{11,12}$ However, it is also known that dietary factors might be important in the pathogenesis of IBS ${ }^{3}$ and the same inflammatory cells, together with eosinophils, are the immunologic effectors of food hypersensitivity (FH). ${ }^{13-17}$

As many symptoms of IBS are common to $\mathrm{FH}-$ abdominal pain and/or discomfort, bloating, alteration of bowel habitsdifferential diagnosis between these 2 conditions is difficult.

In this study involving IBS patients, we aimed to: (1) evaluate the frequency of FH diagnosis; and (2) investigate the diagnostic accuracy of 3 fecal markers-calprotectin, tryptase, and eosinophil cationic protein-in FH diagnosis.

\section{Methods}

One hundred sixty patients (127 females, 33 males, age range $18-60$ years, median age 33 years), who had been consecutively referred for IBS as outpatients to 2 internal medicine clinics (University Hospital of Palermo and Ospedali Civili Riuniti of Sciacca, both in Italy) from February 2007 to June 2009, completed this study.

The inclusion criteria were identical to those recently used ${ }^{18}$ : (1) age $>17$ years, (2) no previous referral to our clinics, and (3) diagnosis of IBS. Patients with a diagnosis of organic gastrointestinal disease were excluded.

Abbreviations used in this paper: $\mathrm{CV}$, coefficient of variation; DBPC double-blind placebo-controlled oral food challenge; ECP, eosinophil cationic protein; $\mathrm{FH}$, food hypersensitivity; HLA, human leukocyte antigen; IBS, irritable bowel syndrome; PBS, phosphate-buffered saline; tTG, tissue transglutaminase. 
IBS diagnosis was based on the Rome II criteria for functional gastrointestinal disorders ${ }^{19}$ and organic gastrointestinal disorders were excluded by an accurate work-up (see Supplementary Methods).

After inclusion, the patients underwent a clinical evaluation, which included a detailed family and personal clinical history and a physical examination. A predesigned questionnaire, regarding the type and severity of the symptoms, was administered to all patients.

None of the enrolled patients were taking any medication at the time of the study.

The Ethics Committee of the University Hospital of Palermo approved the study and all patients gave their informed consent to participate.

\section{Healthy and Disease Control Groups}

Two control groups were selected to compare fecal assay values. One was composed of 40 patients with various gastrointestinal diseases ( 30 females, 10 males, age range 18-59 years, median 31 years): celiac disease $(n=16)$, active ileocolonic Crohn's disease $(n=18)$, and intestinal giardiasis $(n=6)$, diagnosed according to standard serologic, endoscopy, and histology criteria. The other group was composed of 50 healthy volunteers (38 females, 12 male, age range 18 - 60 years, median 30 years).

\section{Study Protocol}

After enrollment in the study, the patients completed the "symptom severity" questionnaire (see Supplementary Methods) and underwent serum total and food allergen-specific IgE determination, together with the fecal assays. The diagnostic tests were performed by different operators, unaware of the clinical history of the patients and the results of the other tests.

The study design was identical to one recently described. ${ }^{18}$ In brief, patients were observed for a 4-week run-in period. After this they underwent a 4-week elimination diet without cow's milk and its derivatives, wheat, egg, tomato, and chocolate. Patients self-reporting FH were also asked to avoid ingestion and/or contact with the food(s) causing symptoms. The patients wrote a dietary diary and adherence to the elimination diet was evaluated by trained dieticians. Patients who referred a symptom and/or sign improvement after the elimination diet period underwent a double-blind placebo-controlled oral food challenge (DBPC), first with cow's milk proteins and then with wheat proteins. ${ }^{20}$

After $\mathrm{FH}$ had been excluded or confirmed, all IBS patients were invited to continue the follow-up with regular visits every 6 months for 2 years. Particular attention was paid when excluding celiac disease diagnosis in FH patients (see Supplementary Methods).

\section{Double-Blind Placebo-Controlled Challenges}

DBPC for cow's milk and wheat were performed as previously described ${ }^{18,20}$ (see Supplementary Methods).

\section{Serum Total IgE and Food Allergen-Specific IgE Antibodies}

Serum samples from all patients were collected and analyzed for serum total IgE and food allergen-specific IgE antibodies by using the Phadia CAP-system (Phadia, Uppsala, Sweden). The following common food allergens were tested: egg, cow's milk, soy, peanut, wheat, tomato, and fish. Levels greater than or equal to $0.35 \mathrm{kU} / \mathrm{L}$ (level 1 on the specific $\operatorname{IgE}$ scale) were considered positive. Total IgE was also determined by the same method with a detection limit of $2 \mathrm{kU} / \mathrm{L}$ and an upper limit of $5000 \mathrm{kU} / \mathrm{L}$. The normal limit for total IgE was $100 \mathrm{kU} / \mathrm{L}$.

\section{Preparation of Stool Samples}

Patients were instructed to defecate directly into a polystyrene container (diameter $15 \mathrm{~cm}$, depth $12 \mathrm{~cm}$ ). Stool samples obviously contaminated with urine were excluded from analysis. For details and methods of tryptase, eosinophil cationic protein (ECP) and calprotectin assays, see Supplementary Methods.

\section{Statistical Analysis}

The $\chi^{2}$ test was used for frequency comparison. Range and median values were calculated for each stool assay. Value comparison of the fecal assays was performed using the MannWhitney test. The Wilcoxon rank-sum test was used to compare the symptoms score before and after the diet treatment. Tables were constructed for frequency and percentage. The sensitivity, specificity, and diagnostic accuracy of food-specific $\operatorname{IgE}$ and fecal assays, along with their 95\% confidence intervals, were calculated by standard statistical methods. ${ }^{21}$

To minimize type I errors, $P<.05$ was considered statistically significant. All statistical analyses were performed using the SPSS 11.0 statistical package (Systat Software, Inc, Chicago, IL).

\section{Results}

During the study period, a total of 198 patients fulfilled the Rome II criteria for IBS diagnosis, but the clinical work-up demonstrated that 38 of them suffered from organic diseases (15 with celiac disease, 10 lactose intolerance, 5 small bowel bacterial overgrowth, 3 large colon adenoma $>3 \mathrm{~cm}$ and high degree dysplasia, 3 colon cancer, and 2 intestinal giardiasis).

After the 4 weeks of run-in observation period, the remaining 160 patients underwent elimination diet. Table 1 summarizes the number of patients with an improved (score reduction $>50$ ), unchanged or worsened (score increase $>50$ ) symptom score on elimination diet. In the 90 patients with unchanged or

Table 1. Number and Percentage of IBS Patients With Scores Improved, Unchanged, or Worsened During a 4-Week Period of Elimination Diet

\begin{tabular}{lccc}
\hline & $\begin{array}{c}\text { Patients } \\
\text { improved }\end{array}$ & $\begin{array}{c}\text { Patients } \\
\text { unchanged }\end{array}$ & $\begin{array}{c}\text { Patients } \\
\text { worsened }\end{array}$ \\
\hline $\begin{array}{l}\text { Number and } \\
\text { percentage }\end{array}$ & $70(44 \%)$ & $61(38 \%)$ & $29(18 \%)$ \\
$\begin{array}{l}\text { Score at baseline } \\
\text { Score during the diet }\end{array}$ & $330 \pm 80^{a}$ & $325 \pm 75$ & $315 \pm 75$ \\
\hline
\end{tabular}

NOTE. Symptom severity scores before and after elimination diet are also shown (mean $\pm \mathrm{SD}$ ). Cow's milk and its derivatives, wheat, egg, tomato, and chocolate, were excluded in all subjects. The patients with self-reported food hypersensitivity also avoided food-causing symptoms.

ap $<.0001$ Wilcoxon rank-sum test. 
Confirmation of IBS diagnosis and exclusion of 'organic' diseases
Elimination diet

Four weeks run-in period (symptoms severity questionnaire, serum total and food allergen-specific $\lg E$ determination and fecal assays )
DBPC challenge with cow's milk and wheat proteins

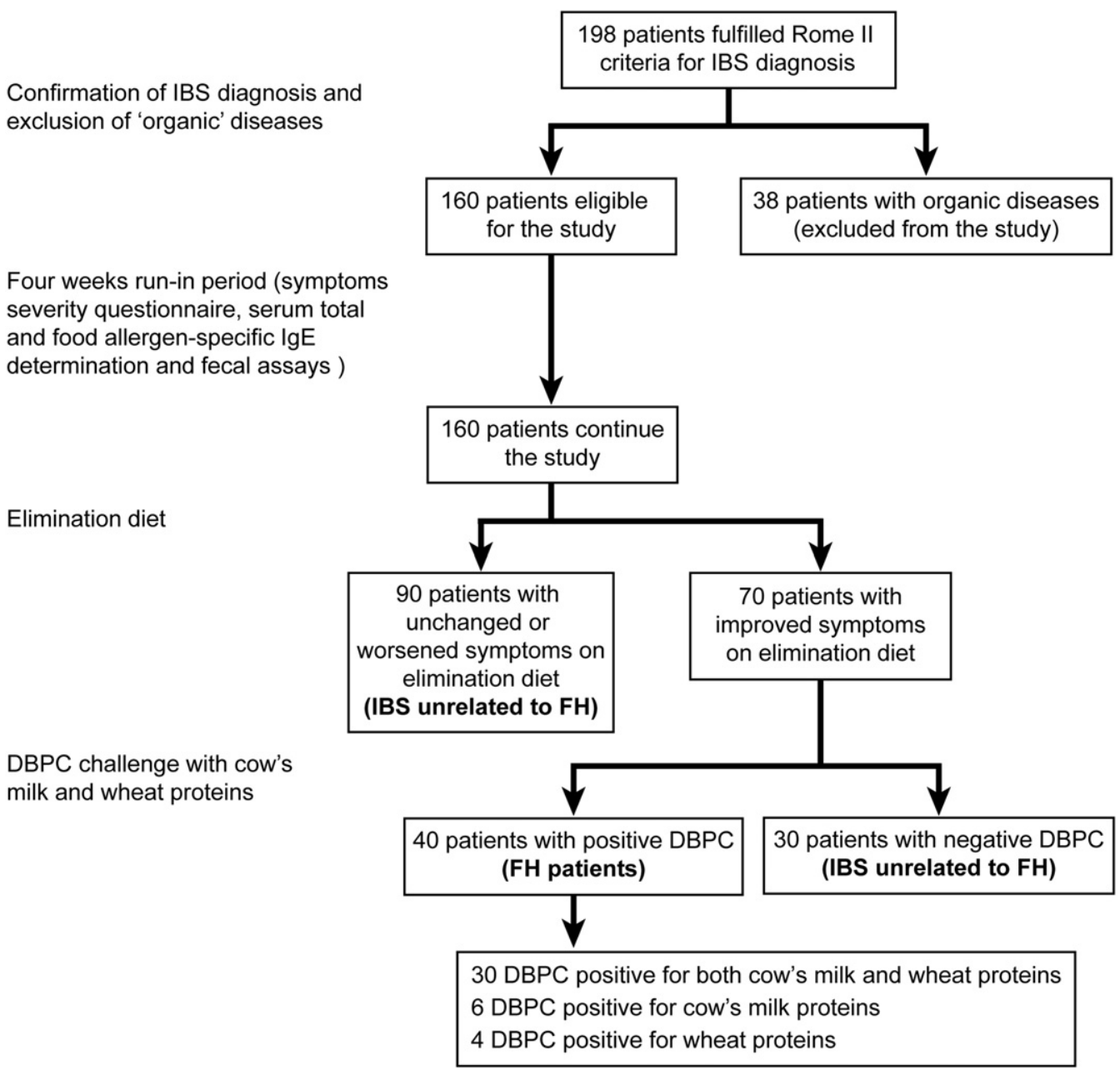

198 patients fulfilled Rome II

criteria for IBS diagnosis

Figure 1. Main results according to the study design. worsened symptoms on elimination diet, a diagnosis of IBS unrelated to FH was made.

The other 70 patients showing a significant reduction in symptom score on elimination diet underwent DBPC challenges. Thirty patients were positive for both cow's milk and wheat protein challenges, 6 were positive exclusively for cow's milk challenge, and 4 were positive exclusively for wheat challenge. In total, 40 patients were positive for DBPC food challenges. In all DBPC-positive patients, symptoms (abdominal pain, bloating, diarrhea, constipation, etc) reappeared after a median period of 2.5 days (range 1- 8 days) after commencing the challenge with cow's milk or wheat proteins. Twenty-four of these 40 patients did not complete the challenge period with the "active food" due to the severity of symptoms. None of these patients reacted on placebo administration.

The 40 patients with positive DBPC challenges to cow's milk and/or wheat proteins fulfilled the Rome II criteria for IBS diagnosis and were diagnosed with $\mathrm{FH}$.

The other 30 patients, who improved on elimination diet, did not react to the DBPC challenges; these subjects also underwent open challenges with other foods and did not react. Consequently, they were diagnosed with IBS not related to FH.

Of the whole group composed of 160 IBS patients, 40 subjects $(25 \%)$ had a final diagnosis of $\mathrm{FH}$ and significantly improved or were cured on the elimination diet.
Figure 1 summarizes the results, according to the study design.

According to the results of the elimination diet and the subsequent DBPC challenges, the patients were then divided into 2 subgroups: group A $(\mathrm{FH})$, including the subjects with scores improved on elimination diet and positive to DBPC challenge with cow's milk and/or wheat proteins $(n=40)$ and group B (IBS not related to $\mathrm{FH}$ ), including the subjects with scores unchanged or worsened on elimination diet and the patients with negative DBPC challenge for cow's milk and wheat proteins $(\mathrm{n}=120)$.

Patients of group A and group B did not differ for sex, age, symptom severity score at entry to the study, clinical characteristics (frequency of predominant diarrhea or constipation or alternating bowel habits), or frequency of associated atopy. However, the duration of IBS symptoms was significantly higher in patients with FH (mean \pm SD: $11.4 \pm 4.2$ vs $6.4 \pm 5.2$ years; $P<.001)$. Furthermore, the $\mathrm{FH}$ patients showed a higher frequency of self-perceived food intolerance (32 of 40 vs 24 of $120 ; P=.01)$ and of history of food hypersensitivity during infancy (10 of 40 vs 9 of 120; $P=.02$ ). Twenty-two of forty (55\%) FH patients showed the DQ2 or DQ8 haplotypes.

Figure 2 shows median and first and third quartile values of fecal tryptase in the patients of group A and group B, as well as in the 2 control groups ("healthy" and "disease" controls). 


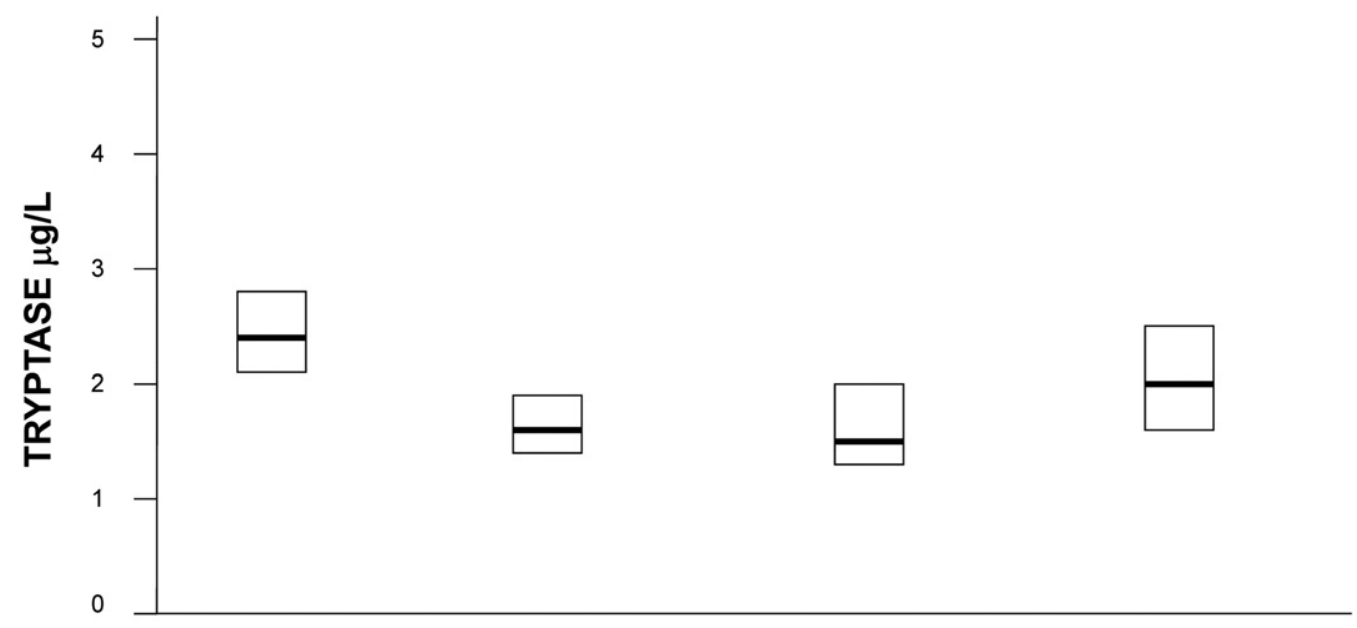

FH

IBS not-FH Healthy controls Disease controls

Figure 2. Fecal tryptase values in 40 patients suffering from FH, in 120 patients with IBS not related to $\mathrm{FH}$, in 50 healthy controls, and in 40 control subjects suffering from different gastrointestinal diseases (disease controls).

Tryptase values were significantly higher in $\mathrm{FH}$ patients than in patients with IBS not related to FH $(P<.001)$ and healthy controls $(P<.001)$; whereas there was no difference between FH patients and "disease" controls. Patients with IBS not related to FH had tryptase values significantly higher than healthy controls $(P<.02)$ and significantly lower than "disease controls" $(P<.002)$.

A similar pattern was seen for ECP (Figure 3). Values were significantly higher in FH patients than in IBS patients not related to $\mathrm{FH}(P<.0001)$ and healthy controls $(P<.0001)$; "disease controls" showed values significantly higher than $\mathrm{FH}$ patients. Patients with IBS not related to FH had ECP values similar to healthy controls and significantly lower than "disease controls" $(P<.0001)$.

As regards calprotectin values, there was no difference between IBS patients with FH and those with IBS unrelated to
FH, nor between these 2 groups and "healthy" controls. However, "disease controls" showed values significantly higher than the other groups.

No differences in the values of the 3 fecal markers were found between patients with predominant constipation and patients with predominant diarrhea within group A and group B (see Supplementary Table 1).

Table 2 shows the number of positive and negative results of the immunologic assays in the IBS patients and in the healthy and "disease" controls. According to these results, Table 3 shows the sensitivity, specificity, and diagnostic accuracy of the immunologic assays in the diagnosis of $\mathrm{FH}$ in patients with a clinical presentation of IBS. Fecal ECP assay showed the highest sensitivity, specificity, and diagnostic accuracy among the assays performed. ECP sensitivity was higher than those of tryptase $\left(\chi^{2} 7.2 ; P=.01\right)$ and calprotectin $\left(\chi^{2} 6 ; P=.02\right)$. ECP

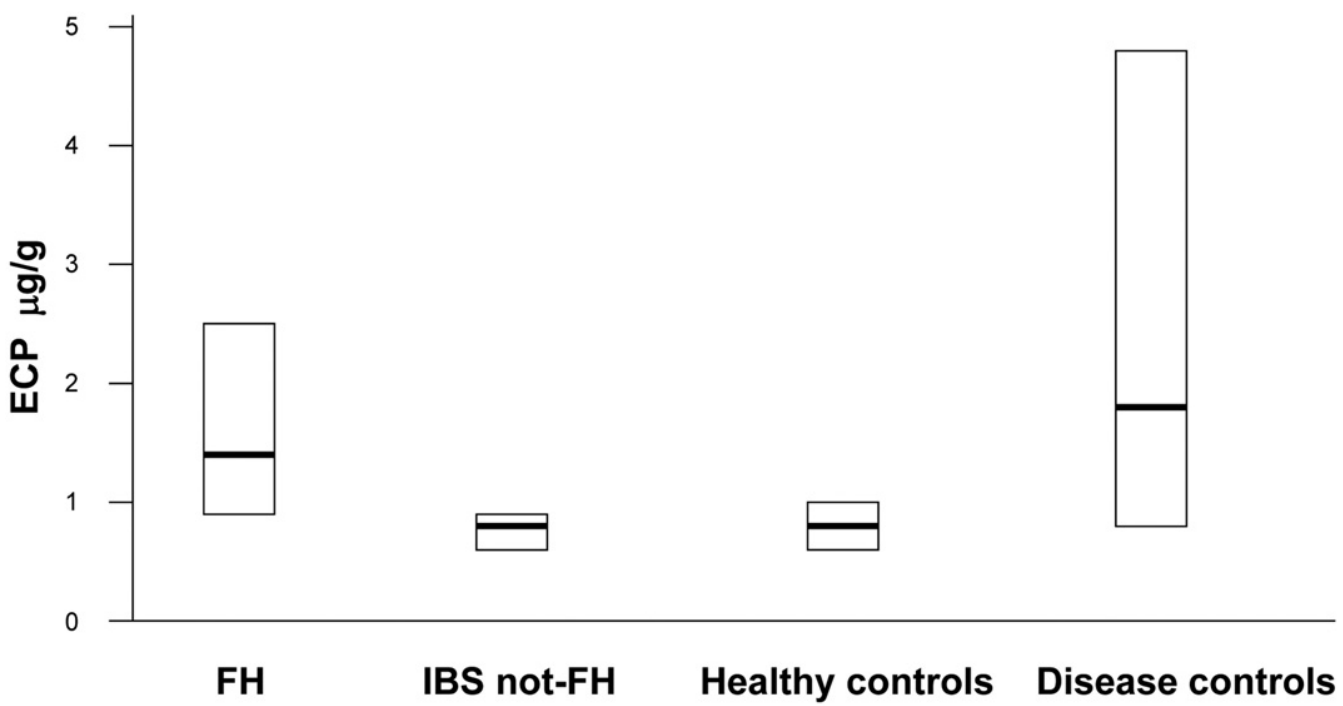

Figure 3. Fecal ECP values in 40 patients suffering from FH, in 120 patients with IBS not related to $\mathrm{FH}$, in 50 healthy controls, and in 40 controls suffering from different gastrointestinal diseases (disease controls). 
Table 2. Results of the Fecal Assays and of Serum Specific IgE in the Patients With IBS Unrelated to $\mathrm{FH}(\mathrm{n}=$ 120), in Those With FH $(n=40)$, in the Healthy Controls $(n=50)$, and in the "Disease" Controls $(n=40)$

\begin{tabular}{|c|c|c|c|c|}
\hline & Tryptase & ECP & Calprotectin & $\begin{array}{c}\text { Serum-specific } \\
\text { IgE }\end{array}$ \\
\hline \multicolumn{5}{|c|}{ IBS unrelated to $\mathrm{FH}$} \\
\hline Positive & 23 & 11 & 22 & 24 \\
\hline Negative & 97 & 109 & 98 & 96 \\
\hline \multicolumn{5}{|c|}{ IBS related to $\mathrm{FH}$} \\
\hline Positive & 13 & 26 & 14 & 24 \\
\hline Negative & 27 & 14 & 26 & 16 \\
\hline \multicolumn{5}{|c|}{$\begin{array}{l}\text { Healthy control } \\
\text { group }\end{array}$} \\
\hline Positive & 2 & 0 & 1 & 0 \\
\hline Negative & 48 & 50 & 49 & 40 \\
\hline \multicolumn{5}{|c|}{$\begin{array}{l}\text { Disease control } \\
\text { group }\end{array}$} \\
\hline Positive & 14 & 29 & 31 & 8 \\
\hline Negative & 26 & 11 & 9 & 32 \\
\hline
\end{tabular}

NOTE. Reference values were: tryptase $<2.4 \mu \mathrm{g} / \mathrm{L}$; ECP $<1 \mu \mathrm{g} / \mathrm{g}$; calprotectin $<50 \mathrm{~g} / \mathrm{kg}$ food allergen-specific IgE antibodies $<0.35$ $\mathrm{kU} / \mathrm{L}$. The following antigens were used in the serum specific IgE assay: alpha-lactalbumin, beta-lactoglobulin, casein, wheat proteins. Positivity for 1 of them was considered as "positive assay," negativity for all was considered as "negative assay."

specificity was high: $91 \%$ in the IBS group and no false positive results among healthy subjects; it was significantly higher than those of tryptase $\left(\chi^{2} 4 ; P=.05\right)$, calprotectin $\left(\chi^{2} 3.9 ; P=.05\right)$, and food-specific $\operatorname{IgE}\left(\chi^{2} 3.9 ; P=.05\right)$. However, high values of ECP were also found in the "disease" controls, mainly in patients with chronic inflammatory bowel disease and intestinal parasitosis.

It was noteworthy that 8 of the $16 \mathrm{FH}$ patients negative for serum specific IgE showed elevated fecal ECP values.

All patients included in the study were followed up for a 2 -year period, during which they were reassessed at intervals of 6 months. FH patients continued to follow an elimination diet with the exclusion of the foods causing the IBS symptoms and all referred the persistent disappearance of symptoms or a consistent improvement. Symptom score at entry to the study was $365 \pm 75$, at 6 months $170 \pm 55$, at 12 months $160 \pm 45$, at 18 months $140 \pm 60$, at 24 months $145 \pm 45$. Occasional ingestion of the eliminated foods always caused the recurrence of the IBS symptoms. Furthermore, all patients referred multiple hypersensitivity to other foods, which we evaluated with elimination diets and open challenges. Apart from cow's milk and wheat, the following foods caused IBS-like symptoms: egg
(18 cases), tomato (14 cases), soy (5 cases), yeast ( 6 cases), pork ( 3 cases), prawn ( 4 cases), fish ( 5 cases), celery ( 3 cases), olive ( 2 cases), nuts (3 cases), carrot ( 2 cases), and meat ( 3 cases).

During the follow-up, some patients underwent further examinations: colonoscopy (5 cases), esopho-gastroscopy and duodenal biopsies (8 cases) - which were all negative. Serum assays for celiac disease (anti-tissue transglutaminase [tTG] and anti-endomisyum antibodies) were regularly repeated every 6 months in all FH patients and were always negative.

Ninety-nine of the IBS patients unrelated to FH completed the 2-year follow-up and 21 were lost. In most of these subjects IBS symptoms persisted periodically, despite several treatments. None of them were found to be suffering from an "organic" cause of symptoms.

\section{Discussion}

IBS is a complex disease and genetic, neurobiological, and psychosocial factors are involved in its pathogenesis. Recently, the role of microscopic inflammation and immune activation in the intestinal mucosa has been elucidated. ${ }^{3,5,22}$ Gastrointestinal infections are more likely to initiate IBS-like symptoms ${ }^{23}$ inducing mucosal inflammation. Impaired intestinal permeability ${ }^{24}$ and a role for mucosal inflammation in regulating the gut-brain axis ${ }^{25}$ have also been reported.

However, it is well known that both mucosal inflammation and impaired intestinal permeability can be due to food hypersensitivity; ${ }^{26}$ furthermore, many patients suffering from IBS report an association of symptoms with specific food ingestion. Numerous recent reports ${ }^{18,27,28}$ have demonstrated that a subgroup of IBS patients suffers from $\mathrm{FH}$ and these subjects improve on elimination diet. However, the relationship between FH and IBS is still considered controversial and it is difficult to distinguish between these conditions because both IBS and FH with gastrointestinal symptoms often have the same clinical presentation. Furthermore, none of the available in vivo and in vitro allergy tests (ie, skin prick test and serum total $\operatorname{IgE}$ and specific IgE assays) have a good diagnostic reliability. ${ }^{29}$

In this study we evaluated a large group of IBS patients and, using a rigorous diagnostic approach-based on elimination diet and DBPC challenge-identified those suffering from $\mathrm{FH}$. Forty IBS patients were positive on DBPC challenge with cow's milk and/or wheat proteins and consequently a high percentage (40/160, 25\%) of cases were diagnosed as FH. In these patients, symptom severity significantly regressed on elimination diet, while numerous previous treatments had not been effective. As 34 out of 160 patients (22\%) showed gluten hypersensitivity, it would be interesting to place these subjects in the complex category of gluten sensitivity. ${ }^{30}$ Previous works have demonstrated that gliadin can induce an increased intestinal permeability and innate immune cell activation. However, these studies

Table 3. Sensitivity, Specificity, and Diagnostic Accuracy (95\% Confidence Interval) of the Fecal Assays and Serum FoodSpecific IgE in the Diagnosis of FH in Patients With Clinical Presentation of IBS $(n=160)$

\begin{tabular}{lcccc}
\hline & Fecal tryptase & Fecal ECP & Fecal calprotectin & Food-specific lgE \\
\hline Sensitivity & $33(24-53)$ & $65(50-78)$ & $35(22-50)$ & $60(45-73)$ \\
Specificity & $81(71-89)$ & $91(82-95)$ & $82(72-88)$ & $80(76-90)$ \\
Diagnostic accuracy & $69(59-77)$ & $84(75-90)$ & $70(58-75)$ & $75(66-83)$ \\
\hline
\end{tabular}

NOTE. Data are shown as \%. Statistics: sensitivity of fecal ECP vs tryptase $P<.02$, vs calprotectin $P<.01$, vs specific IgE not significant; specificity of fecal ECP vs tryptase $P<.05$, vs calprotectin $P<.05$, vs specific IgE $P<.05$. 
on animal models observed these effects only in genetically susceptible subjects. ${ }^{31,32}$ In our study, the DQ2 or DQ8 human leukocyte antigen (HLA) haplotypes was present only in 22/ $40(55 \%)$ of $\mathrm{FH}$ subjects, and it is noteworthy that most patients were suffering from both wheat- and cow's milk-protein hypersensitivity. Consequently, other non-DQ2/DQ8 related mechanisms should be investigated.

As regards the fecal assays, the distribution of tryptase and ECP values was very interesting, whereas fecal calprotectin did not seem to be useful in distinguishing between FH and IBS.

The highest tryptase values were seen in $\mathrm{FH}$ subjects with progressively decreasing-and significantly different-levels in IBS and healthy control subjects. It is known that stimulation of mast cells with IgE-dependent or non-IgE-dependent agonists leads to the release of preformed and newly-synthesized inflammatory mediators. Therefore, tryptase, a serine proteinase, released almost exclusively by mast cells, may be suitable as a marker of mast-cell activation in patients with FH. Moreover, we showed that a high concentration of mast cell-derived proteins in the stools is characteristic of IBS, independently of coexistent $\mathrm{FH}^{3,4,7-9}$ and, in fact, these patients showed significantly higher tryptase values than healthy controls. However, our data indicate the first evidence that fecal tryptase levels (and the underlining mast cell infiltration) is more intensive in $\mathrm{FH}$ than in IBS not related to FH. Further studies should clarify whether the elimination diet can improve the previously demonstrated mast cell mucosal inflammation and the neural damage which has been reported in IBS..$^{3,4,7-9}$ In fact, the relationship between $\mathrm{FH}$ and dismotility in pediatric patients has been clearly demonstrated. ${ }^{20,33-35}$

ECP might be as useful in FH patients as it is in patients with respiratory allergic diseases. ${ }^{36}$ In our study, fecal ECP values were 2-fold higher in FH than in IBS patients or in healthy control subjects. Furthermore, IBS patients had ECP values virtually identical to healthy control subjects. Consequently, fecal ECP seems to be a specific marker of intestinal inflammation due to FH. Previous studies on ECP in adults with $\mathrm{FH}$ have included very few patients, prediagnosed as suffering from FH. Magnusson et al. showed an increase in fecal ECP in $13 \mathrm{FH}$ patients after food challenge ${ }^{37}$ and 8 of these patients fulfilled the Rome II criteria of IBS. However, in our study, ECP assay showed an overall sensitivity of $65 \%$ and we found elevated values in the "disease" control group, so in a clinical setting it may not distinguish between other organic disease and food hypersensitivity.

In conclusion, our study demonstrated that a relatively high percentage of IBS patients are actually suffering from $\mathrm{FH}$ and confirmed that this diagnosis is difficult as FH symptoms are very often very delayed. ${ }^{38}$ In these patients tryptase and ECP are among the pivotal mediators of inflammation; consequently, tryptase and mast cells can be markers of FH rather than of prior gastroenteritis. Fecal assays of ECP may be useful in identifying $\mathrm{FH}$ patients among IBS-presenting subjects.

\section{Supplementary Material}

Note: To access the supplementary material accompanying this article, visit the online version of Clinical Gastroenterology and Hepatology at www.cghjournal.org, and at doi:10.1016/ j.cgh.2011.07.030.

\section{References}

1. Longstreth GF, Hawkey CJ, Mayer EA, et al. Characteristics of patients with irritable bowel syndrome recruited from three sources: implications for clinical trials. Aliment Pharmacol Ther 2001; 15:959-964.

2. Mayer EA. Clinical practice. Irritable bowel syndrome. N Eng J Med 2008;358:1692-1699.

3. De Giorgio R, Barbara G. Is irritable bowel syndrome an inflammatory disorder? Curr Gastroenterol Rep 2008;10:385-390.

4. Weston AP, Biddle WL, Bhatia PS, et al. Terminal ileal mucosal mast cells in irritable bowel syndrome. Dig Dis Sci 1993;38: 1590-1595.

5. Chadwick VS, Chen W, Shu D, et al. Activation of the mucosal immune system in irritable bowel syndrome. Gastroenterology 2002;122:1778-1783.

6. Salzmann JL, Peltier-Koch F, Bloch F, et al. Morphometric study of colonic biopsies: a new method of estimating inflammatory diseases. Lab Invest 1989;60:847-851.

7. Barbara G, Stanghellini V, De Giorgio R, et al. Activated mast cells in proximity to colonic nerves correlate with abdominal pain in irritable bowel syndrome. Gastroenterology 2004;126:693-702.

8. O'Sullivan M, Clayton N, Breslin NP, et al. Increased mast cells in the irritable bowel syndrome. Neurogastroenterol Motil 2000;12: 449-457.

9. Barbara G, Wang B, Stanghellini V, et al. Mast cell-dependent excitation of visceral-nociceptive sensory neurons in irritable bowel syndrome. Gastroenterology 2007;132:26-37.

10. Cremon C, Gargano L, Morselli-Labate AM, et al. Mucosal immune activation in irritable bowel syndrome: gender-dependence and association with digestive symptoms. Am J Gastroenterol 2009;104:392-400.

11. Spiller RC, Jenkins D, Thornley JP, et al. Increased rectal mucosal enteroendocrine cells, T lymphocytes, and increased gut permeability following acute Campylobacter enteritis and in post-dysenteric irritable bowel syndrome. Gut 2000;47:804-811.

12. Barbara G, De Giorgio R, Deng $Y$, et al. Role of immunologic factors and cyclooxygenase 2 in persistent postinfective enteric muscle dysfunction in mice. Gastroenterology 2001;120:1729_ 1736.

13. Brandtzaeg PE. Current understanding of gastrointestinal immunoregulation and its relation to food allergy. Ann N Y Acad Sci 2002;964:13-45.

14. Shah U, Walker WA. Pathophysiology of intestinal food allergy. AdvPediatr 2002;49:299-316.

15. Crowe SE, Perdue MH. Gastrointestinal food hypersensitivity: basic mechanisms of pathophysiology. Gastroenterology 1992; 103:1075-1095.

16. Brandtzaeg P. Development and basic mechanisms of human gut immunity. Nutr Rev 1998;56:S5-S18.

17. Hogan SP, Mishra A, Brandt EB, et al. A pathological function for eotaxin and eosinophils in eosinophilic gastrointestinal inflammation. Nat Immunol 2001;2:1-8.

18. Carroccio A, Brusca I, Mansueto P, et al. A cytological assay for diagnosis of food hypersensitivity in patients with irritable bowel syndrome. Clin Gastroenterol Hepatol 2010;8:254-258.

19. Thompson WG, Longstreth GF, Drossman DA, et al. Functional bowel disorders and functional abdominal pain. Gut 1999 45(Suppl 2):43-47.

20. Iacono G, Cavataio F, Montalto G, et al. Intolerance of cow's milk and chronic constipation in children. N Engl J Med 1998;339: 1100-1104.

21. Feinstein A. On the sensitivity, specificity and discrimination of diagnostic tests. Clin Pharmacol Ther 1975;17:104-110.

22. Guilarte M, Santos J, de Torres I, et al. Diarrhoea-predominant IBS patients show mast cell activation and hyperplasia in the jejunum. Gut 2007;56:203-209.

23. Gwee KA, Leong YL, Graham C, et al. The role of psychological 
and biological factors in postinfective gut dysfunction. Gut 1999;44:400-406.

24. Alonso C, Guilarte M, Vicario M, et al. Maladaptive intestinal epithelial responses to life stress may predispose healthy women to gut mucosal inflammation. Gastroenterology 2008; 135:163-172.

25. Bercik P, Verdú EF, Foster JA, et al. Role of gut-brain axis in persistent abnormal feeding behavior in mice following eradication of Helicobacter pylori infection. Am J Physiol Regul Integr Comp Physiol 2009;296:R587-R594.

26. Vighi G, Marcucci F, Sensi L, et al. Allergy and the gastrointestinal system. Clin Exp Immunol 2008;153;Suppl 1:3-6.

27. Zar S, Kumar D, Benson MJ. Food hypersensitivity and irritable bowel syndrome. Aliment Pharmacol Ther 2001;15:439-449.

28. Atkinson W, Sheldon TA, Shaath N, et al. Food elimination based on IgG antibodies in irritable bowel syndrome: a randomised controlled trial. Gut 2004;53:1459-1464.

29. Sampson HA, Sicherer SH, Birnbaum AH. AGA technical review on the evaluation of food allergy in gastrointestinal disorders. American Gastroenterological Association. Gastroenterology 2001; 120:1026-1040.

30. Verdu E, Armstrong D, Murray J. Between celiac disease and irritable bowel syndrome: the "no man's land" of gluten sensitivity. Am J Gastroenterol 2009;104:1587-1594.

31. Verdu EF, Huang XX, Natividad J, et al. Gliadin-dependent neuromuscular and epithelial secretory responses in the gut. Am J Physiol Gastrointest Liver Physiol 2008;294:G217-G225.

32. Pinier M, Verdú EF, Nasser-Eddine M, et al. Polymeric binders suppress gliadin-induced toxicity in the intestinal epithelium. Gastroenterology 2009;136:288-298.
33. Carroccio A, Scalici C, Maresi M, et al. Chronic constipation and food intolerance: a model of proctitis causing constipation. Scand J Gastroenterol 2005;40:33-42.

34. Iacono G, Bonventre S, Scalici C, et al. Food intolerance and chronic constipation: manometry and histology study. Eur J Gastroenterol Hepatol 2006;18:143-150.

35. Borrelli O, Barbara G, Di Nardo G, et al. Neuroimmune interaction and anorectal motility in children with food allergyrelated chronic constipation. Am J Gastroenterol 2009;104: 454-463.

36. Bousquet J, Chanez P, Lacoste JY. Eosinophilic inflammation in asthma. N Engl J Med 1990;323:1033-1039.

37. Magnusson J, Gellerstedt M, Ahlstedt S, et al. A kinetic study in adults with food hypersensitivity assessed as eosinophil activation in fecal samples. Clin Exp Allergy 2003;33:10521059.

38. Carroccio A, Montalto G, Custro C, et al. Evidence of very delayed clinical reactions to cow's milk in cow's milk-intolerant patients. Allergy 2000;55:574-579.

Reprint requests

Address requests for reprints to: Antonio Carroccio, MD, Ospedali Civili Riuniti, Sciacca (AG), Italy. e-mail: acarroccio@hotmail.com; fax: +390925 84757 .

Conflicts of interest

The authors disclose no conflicts. 


\section{Supplementary Methods}

\section{Rome II Criteria for IBS Diagnosis}

The included patients had suffered from recurrent abdominal discomfort or pain at least 3 days per month in the previous 3 months, associated with 2 or more of the following: (1) improvement with defecation; (2) onset associated with a change in stool frequency; or (3) onset associated with a change in stool appearance.

\section{Work-up for IBS Diagnosis}

All patients underwent first-step hematology and chemistry tests (including erythrocyte sedimentation rate, serum C-reactive protein, blood cell counts, electrolytes, and thyroid, liver, and renal function), serologic assays for celiac disease (anti-transglutaminase $\operatorname{IgA}$ and $\operatorname{IgG}$ ), stool examination for occult blood, ova and parasites, and a lactose- $\mathrm{H} 2$ breath test. Patients also underwent sigmoidoscopy with biopsy if under 40 or colonoscopy with biopsy if over 40 years of age. Patients with negative results for all of the examinations described above and with a clinical history indicating IBS, according to the Rome II criteria, were considered to be suffering from IBS and were enrolled in the study.

\section{Clinical Follow-up and Methods to Exclude a Diagnosis of Celiac Disease}

Clinical follow-up was performed with regular visits every 6 months for 2 years. During the follow-up visits the patients again underwent physical examination, clinical history, and routine hemato-chemical assays and when considered opportune some instrumental examinations were repeated. Particular attention was paid in excluding celiac disease diagnosis in FH patients.

At the entry in the work-up evaluation, the patients who had regularly eaten wheat underwent serologic assays for celiac disease (anti-transglutaminase IgA and IgG-anti-tTG IgA and $\operatorname{IgG}$ ) and if the results were negative they were included. Patients with a recent history of reduced wheat consumption were instead asked to reintroduce wheat into the diet and anti-tTG IgA and IgG assays were repeatedly performed. Furthermore, these subjects underwent HLA determination to search for DQ2 or DQ8 or the single DQB $1 * 02$ allele. Patients with negative serum anti-tTGs not carrying the alleles for DQ2 or DQ8 or the DQB $1 * 02$ allele were considered not to be suffering from celiac disease. Patients carrying the alleles for DQ2 or DQ8 or the DQB1*02 allele, although negative for serum antitTGs, underwent duodenal biopsy after regular wheat consumption and symptoms reappearance. A normal villi aspect (villi:crypts ratio $>3$ ) and a normal intraepithelial lymphocytes count $(<25$ intraepithelial lymphocytes and/or 100 enterocytes) were considered to exclude a celiac disease diagnosis. Patients with doubtful histology were excluded and re-evaluated during the follow-up.

HLA was also determined in all patients with a definitive diagnosis of gluten sensitivity who had not undergone this assay at the entry in the study.

\section{Symptoms Questionnaire}

Symptoms were assessed using a questionnaire scoring system validated for use in IBS, including an IBS symptom severity score (range $0-500$ ). This is a system for scoring pain, distension, bowel dysfunction, and general well-being, with mild, moderate, and severe cases indicated by scores of 75 to 175,175 to 300 , and $>300$, respectively. A reduction in score of 50 or more was regarded as a clinically significant improvement, whereas an increase in score of 50 or more was considered as a clinically significant worsening. ${ }^{21}$

\section{Double-Blind Placebo-Controlled Challenges}

DBPC for cow's milk was performed by administering capsules coded as A or B containing milk proteins (daily dose $11 \mathrm{~g}$, equal to the protein content of $300 \mathrm{~mL}$ of cow's milk) or xylose, respectively. DBPC for wheat proteins was performed with capsules coded as $\mathrm{C}$ or D containing wheat (daily dose $13 \mathrm{~g}$, equal to the protein content of $100 \mathrm{~g}$ of bread) or xylose, respectively. Capsules A or B were given for 2 consecutive weeks, and then after 1 week of washout the patients received the other capsules for another 2 weeks. After 1 week of washout, capsules $\mathrm{C}$ or $\mathrm{D}$ were given for 2 consecutive weeks, then after another week of washout, the patients received the other capsules for 2 weeks. The challenges were stopped when a clinical reaction occurred, ie, the onset of abdominal discomfort or pain, associated with a change in stool frequency and/or appearance.

\section{Preparation of Stool Samples (Further Details)}

An aliquot of $1 \mathrm{~g}$ was separated and stored at $-80^{\circ} \mathrm{C}$ until analysis. After thawing, overnight in the refrigerator or at room temperature using a fan, approximately $100 \mathrm{mg}$ of feces were taken and diluted in an extraction buffer (weight:volume ratio 1:50), consisting of phosphate-buffered saline (PBS), supplemented with $12 \mathrm{mmol} / \mathrm{L}$ ethylenediamine tetraacetic acid, $0.1 \% \mathrm{~N}$-cetyl-trimethyl ammonium bromide, $20 \%$ glycerol, $0.005 \%$ Tween 20 , and $1 \%$ bovine serum albumin, and a protease inhibitor cocktail (Sigma-Aldrich, Saint Louis, Missouri, USA), containing $4 \mathrm{mmol} / \mathrm{L}$ 4-(2-aminoethyl)-benzensulfonyl fluoride, $0.26 \mathrm{mmol} / \mathrm{L}$ bestatin, $28 \mu \mathrm{mol} / \mathrm{L} \mathrm{E-28,} 2 \mu \mathrm{mol} / \mathrm{L}$ leupeptin, and $0.6 \mu \mathrm{mol} / \mathrm{L}$ aprotinin, $\mathrm{pH}$ 7.4. The mixture was then homogenized using a Polytron mixer (Glen Mills, Inc., Clifton, New Jersey) until a homogenous solution was obtained (approximately 10-90 seconds). The homogenate (1 mL) was centrifuged at $20,000 \mathrm{~g}$ for 30 minutes and the clear extract supernatant $(0.5 \mathrm{~mL})$ collected and frozen at $-20^{\circ} \mathrm{C}$ for later measurement.

\section{Fecal Markers Assays}

Measurement fecal tryptase. Fecal tryptase concentration was analyzed by immunoassay performed in duplicate using the ImmunoCAP system (Pharmacia Diagnostics, Uppsala, Sweden) according to the manufacturer's instructions. The fluorescence of the samples was always adjusted for the fluorescence of the diluent before evaluation. The measurement range of the assay was $<1$ to $200 \mu \mathrm{g} / \mathrm{L}$ and the upper normal limit was considered equal to the highest value recorded in over 100 healthy subjects in our laboratory: $2.4 \mu \mathrm{g} / \mathrm{L}$. Coefficients of variation, based on 3 control samples, analyzed in duplicate, on 18 occasions, were between $1.8 \%$ and $3.6 \%$ within assays and between $6.4 \%$ and $10 \%$ between assays.

Measurement of fecal ECP. The fecal levels of ECP were measured using the Immulite 2000 (Siemens Medical Solutions Diagnostics, Tarrytown, New York), according to the manufacturer's instructions. The optimal sample dilution for 
assay was first examined. The extraction buffer did not interfere with the measurements by immunoassay. Samples diluted at 1:40 or more with PBS showed good linearity of the assay system, whereas samples diluted at 1:20 with PBS showed a higher level than theoretically expected. This was considered to be due to possible interference present in the stool. Therefore, the samples were diluted at $1: 40$ or more with PBS. The measurement range of the assay was 0.4 to $70 \mu \mathrm{g} / \mathrm{g}$ and the upper normal limit was considered equal to the highest value recorded in over 100 healthy subjects in our laboratory: $1 \mu \mathrm{g} / \mathrm{g}$. Intra-assay coefficient of variation (CV) was $9.1 \%$, interassay CV was $12.5 \%$.

Measurement of calprotectin. Fecal calprotectin was measured by a commercially available quantitative enzymelinked immunoassay (Calprest, Eurospital, Trieste, Italy). The supernatants were diluted 1:50 and $100 \mathrm{~mL}$ of each sample were added to the wells of a microtiter plate and incubated at room temperature for 45 minutes. After the plate was washed 4 times with diluted washing solution, $100 \mathrm{~mL}$ of purified rabbit anticalprotectin antibodies conjugated with alkaline phosphatase were added and incubated for 45 minutes at room temperature. A second washing procedure was performed and $100 \mathrm{~mL}$ of enzyme substrate solution were added to each well and the optical density was read at $405 \mathrm{~nm}$ by a single biologist unaware of the clinical diagnosis. Calprotectin concentration was calculated from the standard curve obtained with the kit standards and expressed as $\mathrm{mg} / \mathrm{g}$ of stool.

According to manufacturer's instructions the cutoff point was $50 \mathrm{~g} / \mathrm{kg}$.

Intra-assay $\mathrm{CV}$ calculated on 20 samples was $5.2 \%$, interassay CV was $7.1 \%$. 
Supplementary Table 1. Results of the Fecal Markers (Mean \pm SD) in the Whole Study Group

\begin{tabular}{lcccc}
\hline & d-IBS & c-IBS & a-IBS & \\
\hline ECP values $(\mu g / g)$ & $1.0 \pm 1.1$ & $1.2 \pm 1.3$ & $1.2 \pm 1.6$ & Not significant \\
Tryptase values $(\mu g / L)$ & $1.3 \pm 0.9$ & $1.4 \pm 0.8$ & $1.2 \pm 0.9$ & Not significant \\
Calprotectin values $(g / \mathrm{kg})$ & $33 \pm 29$ & $43 \pm 39$ & $32 \pm 32$ & Not significant \\
\hline
\end{tabular}

NOTE. The whole study group consisted of patients with IBS not related to FH plus FH patients, subdivided according the frequency of the bowel movements: diarrhea-predominant IBS, constipation predominant IBS, and alternating bowel movements IBS. 